\title{
Polarization Dependent Pulse Distortion in Stimulated Brillouin Scattering Slow Light Systems
}

\author{
Avi Zadok ${ }^{1}$, Sanghoon Chin ${ }^{2}$, Elad Zilka ${ }^{3}$, Avishay Eyal ${ }^{3}$, Luc Thévenaz ${ }^{2}$, and Moshe Tur ${ }^{3}$ \\ ${ }^{1}$ Department of Applied Physics, MC 128-95, California Institute of Technology, Pasadena, CA 91125, USA \\ ${ }^{2}$ Ecole Polytechnique Fédérale de Lausanne, Institute of Electrical Engineering, STI-GR-SCI Station 11, 1015 Lausanne, Switzerland \\ ${ }^{3}$ School of Electrical Engineering, Faculty of Engineering, Tel-Aviv University, Tel-Aviv 69978, Israel \\ avizadok@caltech.edu; avishay@eng.tau.ac.il; luc.thevenaz@epfl.ch; tur@eng.tau.ac.il;
}

\begin{abstract}
Stimulated Brillouin scattering slow light delay is shown to introduce an inherent polarization mode dispersion, which can dominate the broadening and distortion of signal pulses. The effect is demonstrated in both simulations and experiments.

(C) 2009 Optical Society of America

OCIS Codes: (290.5900) Scattering, Stimulated Brillouin; (120.5410) Polarimetry; (999.9999) Slow and Fast Light
\end{abstract}

\section{Introduction}

Stimulated Brillouin scattering (SBS) is underlying many fiber-based slow and fast light setups [1,2]. As SBS is driven by interference between the pump and signal waves, the signal gain is known to be strongly and inherently dependent on polarization [3]. In a recent work, a vector formalism for SBS amplification in the presence of arbitrary fiber birefringence was provided [4]. The analysis showed that in the undepleted pump regime, the SBSamplifying fiber is equivalent to a linear medium with a polarization dependent gain. The maximum and minimum gains in that medium are associated with a pair of orthogonal states of polarization (SOPs) of the signal wave, which are determined by the pump input SOP and the birefringence properties of the fiber [4]. SBS amplification in randomly birefringent fibers was also recently examined in $[5,6]$. Most previous works, however, have dealt with continuous wave signals [3-5]. The work in [6], although dedicated to the delay of signal pulses, did not address the pulse distortion.

In the linear propagation regime, chromatic dispersion and polarization mode dispersion (PMD) are long known to cause broadening and distortion of pulses [7]. In SBS slow-light setups, pulse distortion due to the limited bandwidth and the dispersion associated with the scalar frequency dependence of SBS has also been thoroughly documented [8]. In this work, we show that the vector nature of SBS gives rise to polarization-related distortion, which is yet another mechanism responsible for pulse broadening in slow light setups. A pulse aligned with the maximum gain polarization undergoes a longer delay then that of a pulse adjusted for the minimum gain. Consequently, there is a differential group delay between two orthogonal components of an arbitrarily polarized pulse. The distortion is analogous but different from that of linear birefringence PMD, with the orthogonal SOPs of maximum and minimum gain taking up a similar role to that of the principal axes [7]. The broadening associated with SBS-related polarization distortion can be significant. For example, we show that an arbitrarily polarized input signal pulse, subject to moderate SBS gain, can become broader then a pulse aligned for maximum delay and amplification. The effect is demonstrated in numerical simulations and experiments of SBS slow light delay.

\section{Analysis: polarization mode dispersion introduced by SBS amplification and delay of pulses}

The propagation equation for a single Fourier component of the signal Jones vector, $\vec{E}_{\text {sig }}(z, \Delta \omega)$, subject to both SBS and birefringence, is given by [4]:

$$
\frac{\mathrm{d} \vec{E}_{\text {sig }}(z, \Delta \omega)}{\mathrm{d} z}=\left[\frac{\mathrm{d} \mathbf{T}(z)}{\mathrm{d} z} \mathbf{T}^{-1}(z)+\frac{\gamma_{0}(\Delta \omega)}{2} \vec{E}_{\text {pump }}(z) \vec{E}_{\text {pump }}^{\dagger}(z)\right] \vec{E}_{\text {sig }}(z, \Delta \omega)
$$

Here $z$ denotes position along a fiber of length $L, \Delta \omega$ is the frequency detuning of the particular wave component from the frequency of maximum SBS amplification, and $\vec{E}_{\text {pump }}(z)$ is the Jones vector of an undepleted pump wave. The pump wave is assumed to be monochromatic for simplicity, even though the following analysis would hold for a broadened, undepleted pump. The complex SBS gain coefficient $\gamma(\Delta \omega)$ is of Lorenzian shape: $\gamma(\Delta \omega)=\gamma_{0} /\left(1+j 2 \cdot \Delta \omega / \Gamma_{B}\right)$, where $\gamma_{0}[\mathrm{~W} \cdot \mathrm{m}]^{-1}$ denotes the peak gain coefficient of SBS and $\Gamma_{B} / 2 \pi \approx 30 \mathrm{MHz}$ is the SBS linewidth [1]. The energy conserving term $[\mathrm{d} \mathbf{T}(z) / \mathrm{d} z] \mathbf{T}^{-1}(z)$, which describes the local birefringence, is 


\section{PDPC1.pdf}

virtually frequency independent within the narrow $\Gamma_{B}$, implying that the linear PMD of the fiber can be ignored. For simplicity, we leave out the linear losses in the typically short fiber.

Since Eq. (1) is linear, we may examine the propagation of signal pulses via Fourier component analysis. In a previous study [4], we obtained the following expression for a single-frequency signal Jones vector at the fiber output:

$$
\vec{E}_{s i g}(L, \Delta \omega)=\mathbf{U}(\Delta \omega) \cdot\left[\begin{array}{cc}
G_{\max }(\Delta \omega) & 0 \\
0 & G_{\min }(\Delta \omega)
\end{array}\right] \cdot \mathbf{V}^{\dagger}(\Delta \omega) \cdot \vec{E}_{s i g}(0, \Delta \omega),
$$

where $\mathbf{U}, \mathbf{V}$ are unitary matrices and $G_{\max }, G_{\min }$ denote the maximum and minimum amplitude gain functions, respectively. $G_{\max }, G_{\min }$ are associated with a pair of orthogonal input unit signal vectors, $\hat{e}_{s i g}^{i_{n} \max }(\Delta \omega)=\mathbf{V} \cdot\left[\begin{array}{ll}1 & 0\end{array}\right]^{T}$ and $\hat{e}_{s i g}^{i_{\text {I }} \min }(\Delta \omega)=\mathbf{V} \cdot\left[\begin{array}{ll}0 & 1\end{array}\right]^{T}$ respectively, and with a pair of orthogonal output unit vectors, $\hat{e}_{s i g}^{\text {out } \max }(\Delta \omega)=\mathbf{U} \cdot\left[\begin{array}{ll}1 & 0\end{array}\right]^{T}$ and $\hat{e}_{\text {sig }}^{\text {out } \min }(\Delta \omega)=\mathbf{U} \cdot\left[\begin{array}{ll}0 & 1\end{array}\right]^{T}$ respectively.

We now proceed to examine the spectral properties of Eq. (2). A key observation, supported by both simulation and experiment, is that although $G_{\max }, G_{\min }$ vary exponentially with frequency through $\gamma(\Delta \omega), \hat{e}_{s i g}^{i_{2} \max }(\Delta \omega)$ and $\hat{e}_{s i g}^{i i_{\text {m }} \text { min }}(\Delta \omega)$, as well as their output counterparts, are nearly frequency independent. This behavior is illustrated in the simulations results of Fig. 1(a), which show the unit three-element Stokes space representation $\hat{S}_{s i g}^{i_{n} \text { min }}(\Delta \omega)$ of $\hat{e}_{s i g}^{i_{i} \min }(\Delta \omega)$ for a particular fiber realization, calculated directly using Eq. (1). The inner product $\hat{s}_{s i g}^{\text {in } \min }(\Delta \omega)^{\dagger} \cdot \hat{s}_{s i g}^{i_{\text {in }} \min }(0)$, in the particular example, always exceeded 0.94 . Stokes space projections $\hat{S}_{s i g}^{i_{\text {I }} \min }(\Delta \omega)^{\dagger} \cdot \hat{S}_{s i}^{i_{\text {I }} \min }(0)>0.95$ were also measured in an auxiliary experiment, using the technique described in [4], for both a $2250 \mathrm{~m}$ long fiber (pump power of $20-35 \mathrm{~mW}$ ) and a $415 \mathrm{~m}$ long fiber $(150 \mathrm{~mW}$ ). This property of SBS in birefringent fibers is in compliance with previous results [4], which showed that $\hat{e}_{s i g}^{i_{n} \text { max }}(0), \hat{e}_{s i g}^{i_{n} \min }(0)$ are nearly pump power invariant.

Given the aforementioned property of SBS in birefringent fibers, it is possible to replace Eq. (2) with a pair of decoupled, scalar SBS amplification equations, one associated with the frequency domain gain function $G_{\max }(\Delta \omega)$ and the input SOP $\hat{e}_{s i g}^{i_{n} \max }(0)$, and the other with $G_{\min }(\Delta \omega)$ and $\hat{e}_{s i g}^{i_{i} \min }(0)$. We may conveniently examine the propagation of an arbitrarily polarized input signal pulse using decomposition in the basis of $\hat{e}_{s i i_{s}}^{i_{\text {max }}}(0)$ and $\hat{e}_{s i g}^{i_{\text {n }} \min }(0)$. Since typically $G_{\max }>>G_{\min }$, we expect that the SBS induced delay of a pulse aligned with $\hat{e}_{s i g}^{i_{i} \max }(0)$ will be considerably longer than that of a pulse aligned with $\hat{e}_{s i g}^{i_{n} \text { min }}(0)$. These SOPs, therefore, take up a role similar to that of the principal axes of linear birefringence induced PMD, on top of representing polarization dependent gain axes.

\section{Numerical and experimental results}

SBS-induced PMD was demonstrated through numerical simulations of Eq. (1). Figure 1(b) shows examples of the calculated, normalized signal output power as a function of time $t$, obtained for a signal input Gaussian pulse with a full width at half maximum (FWHM) of $17 \mathrm{~ns}$ and for three different signal input SOPs. The monochromatic pump power was $560 \mathrm{~mW}$ and the fiber was $140 \mathrm{~m}$ long, with a beat length of $40 \mathrm{~m}$ and a birefringence correlation length of $10 \mathrm{~m}$ [9]. We denote the output power of pulses aligned with $\hat{e}_{s i g}^{i_{\text {in }} \max }(0)$ and $\hat{e}_{s i g}^{i_{\text {in }} \min }(0)$ as $P_{s i g}^{\text {out } \max }(t)$ and $P_{s i g}^{\text {out } \min }(t)$, respectively. The SBS gain, group delay and output FWHM of $P_{\text {sig }}^{\text {out } \max }(t)$ were $33 \mathrm{~dB}, 38 \mathrm{~ns}$ and $54.5 \mathrm{~ns}$, respectively. The corresponding values for $P_{\text {sig }}^{\text {oun } \min }(t)$ were $9 \mathrm{~dB}, 7 \mathrm{~ns}$ and $26 \mathrm{~ns}$. Interestingly, only a slight detuning of the input SOP from $\hat{e}_{s i g}^{i_{\text {in } \min }}(0): \hat{e}_{s i g}^{i_{\text {in near }} \text { min }}=\alpha \hat{e}_{s i g}^{i_{\text {in } \max }}(0)+\sqrt{1-|\alpha|^{2}} \hat{e}_{s i g}^{i_{\text {in } \min }}(0)$ with $|\alpha|^{2}=0.002$, led to an output FWHM of 60 ns. This output width is broader than that of the maximally delayed pulse, even though the SBS gain for the particular input SOP was only $9.3 \mathrm{~dB}$ ! The figure also shows an approximate calculation of the output power: $|\alpha|^{2} P_{s i g_{\text {m }} \text { max }}^{\text {oux }}(t)+\left(1-|\alpha|^{2}\right) P_{s i g}^{\text {out } \text { min }_{\text {min }}}(t)$. This first-order approximation is close to the exact solution, with the excessive pulse broadening well accounted for by a first-order representation of SBS-induced PMD. The small deviations between the exact and approximate solutions are due to the weak frequency dependence of $\hat{e}_{s i g}^{i_{n} \max }, \hat{e}_{s i g}^{i_{n} \text { min }}$. 


\section{PDPC1.pdf}

a:

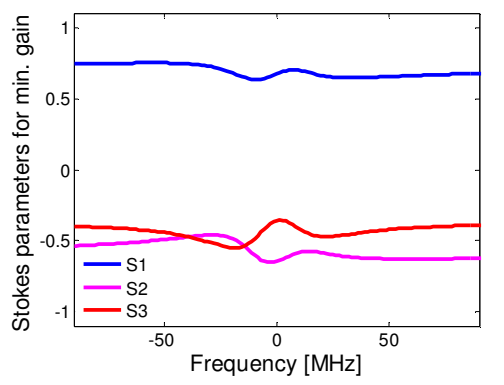

c:

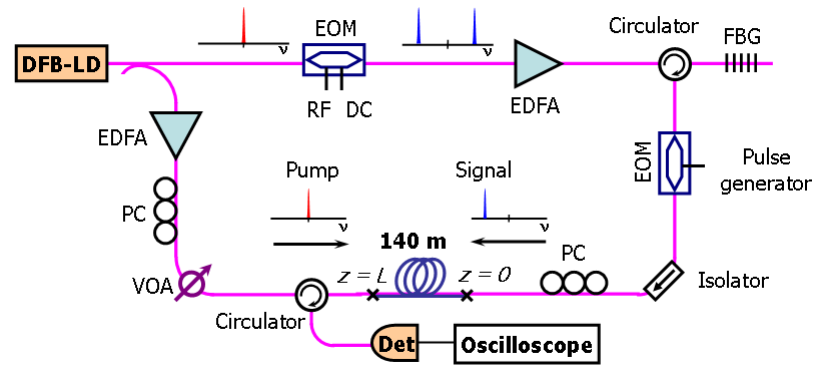

b:

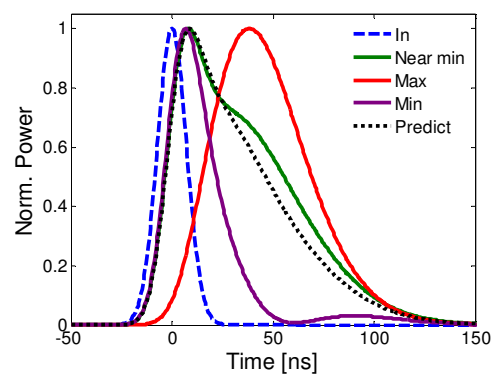

d:

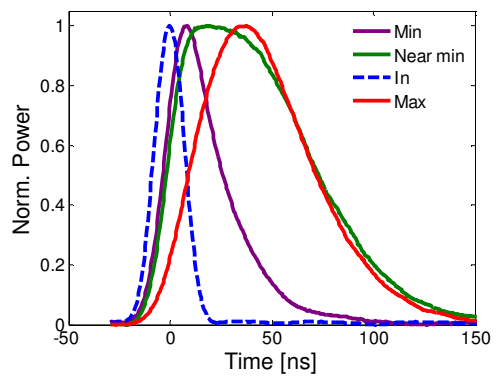

Fig. 1: (a) Calculated Stokes parameters $S_{I}$ (blue), $S_{2}$ (magenta) and $S_{3}$ (red) of the input signal that lead to the minimum SBS gain, as a function of frequency detuning $\Delta \omega$, for a particular fiber realization. The fiber length $L$ was $140 \mathrm{~m}$, with a beat length of $40 \mathrm{~m}$ and a birefringence coupling length of $10 \mathrm{~m}$. The pump power was $560 \mathrm{~mW}$. The SBS gain parameters were $\gamma_{0}=0.16[\mathrm{~W} \cdot \mathrm{m}]^{-1}$ and $\Gamma_{B} / 2 \pi=30 \mathrm{MHz}$. (b) Calculated, normalized signal power as a function of time. Dashed line: input Gaussian pulse with a FWHM of $17 \mathrm{~ns}$. Solid lines: calculated output pulses. The input SOP was aligned for minimum gain (leftmost curve, magenta), slightly detuned from minimum gain ("near minimum", central curve, green), and aligned for maximum gain (rightmost curve, red). Dotted line: approximate output pulse, calculated using a decomposition of the "near minimum" input SOP in the basis of $\hat{e}_{s i}^{i_{\text {i }} \max }(0), \hat{e}_{s i g}^{i_{\text {m }} \text { min }}(0)$. The simulated fiber parameters and the pump power were the same as those of panel (a). (c) Experimental setup for observing SBS-PMD. VOA: variable optical attenuator. Det: detector. RF: radio frequency. DC: direct current. (d) Measured, normalized signal power as a function of time. Dashed line: input Gaussian pulse. Solid lines: output pulses. The input SOP was aligned for minimum (leftmost, magenta), "near minimum" (center, green), and maximum (rightmost, red) gain. The input pulse FWHM, the fiber length and the pump power were the same as those of panel (b).

Excessive polarization-related pulse broadening in an SBS slow light setup was also observed experimentally. The measurement setup is shown in Fig. 1(c) [4]. Light from a distributed feedback laser diode (DFB-LD) was split by a directional coupler. The lower branch was amplified using an erbium-doped fiber amplifier (EDFA), and was launched into the fiber under test as an SBS pump wave at $z=L$. The upper branch was double-sideband modulated at the Brillouin frequency shift of the fiber under test $(10.91 \mathrm{GHz})$, with the bias of the electro-optic modulator (EOM) adjusted to suppress the optical carrier frequency. The upper frequency sideband was discarded by a narrowband fiber Bragg grating (FBG), and the filtered, lower frequency sideband was used as the signal wave at the Stokes frequency. This signal was then modulated by Gaussian pulses, using a second EOM, and was launched into the fiber at $z=0$. A polarization controller (PC) was used to adjust the input signal SOP. The pump power, fiber length and input pulse FWHM were $560 \mathrm{~mW}, 140 \mathrm{~m}$ and $17 \mathrm{~ns}$, as in the simulations.

Figure 1(d) shows measurements of the normalized output power for different input SOPs. A pulse slightly detuned from the SOP of minimum gain, amplified by only $11 \mathrm{~dB}$, was broadened to a FWHM of $75 \mathrm{~ns}$. The maximally delayed pulse, amplified by $21 \mathrm{~dB}$, was broadened to a FWHM of only $63 \mathrm{~ns}$. This polarization dependence of the pulse broadening is not predicted by the scalar model. The results demonstrate that SBS slow light implementations may introduce polarization induced distortions, attributed to the vector nature of SBS.

\section{References}

1. L. Thévenaz, Nature Photonics 2, 474-481 (2008).

2. G. M. Gehring, R. W. Boyd, A. L. Gaeta, D. J. Gauthier, and A. E. Willner, J. Lightwave Technol. 26, 3752-3762 (2008).

3. M. O. van Deventer and A. J. Boot, J. Lightwave Technol. 12, 585-590 (1994).

4. A. Zadok, E. Zilka, A. Eyal, L. Thévenaz, and M. Tur, Opt. Express 16, 21692-21707 (2008).

5. A. Galtarossa, L. Palmieri, M. Santaguistina, L. Schenato, and L. Ursini, IEEE Photon. Technol. Lett. 20, 1420-1422 (2008).

6. D. R. Walker, M. Bashkanski, A. Gulian, F. K. Fatemi, and M. Steiner, J. Opt. Soc. Am. B 25, C61-C64 (2008).

7. J. P. Gordon and H. Kogelnik, P. Natl. Acad. Sci. USA 97, 4541-4550 (2000).

8. Z. Zhu, D. J. Gauthier, Y. Okawachi, J. E. Sharping, A. L. Gaeta, R. W. Boyd, and A. E. Willner, J. Opt. Soc. Am. B 22, 2378-2384 (2005).

9. P. K. A. Wai and C. R. Menyuk, J. Lightwave Technol. 14, 148-157 (1996). 